\title{
Meeting Mandatory Infectious Disease Reporting Requirements: The Department of Veterans Affairs' Plan
}

\author{
Brooke O'Hanley Selzer*, Annette Lin, Gina Oda and Mark Holodniy \\ Office of Public Health Surveillance and Research, Department of Veterans Affairs, Palo Alto, CA, USA
}

\section{Objective}

In June 2013, in anticipation of the passage of proposed federal legislation (S 875 and HR 1792), the Department of Veterans Affairs (VA) issued a Directive requiring mandatory reporting of infectious diseases to various public health authorities (VHA Directive 2013008). In terms of implementation strategies, the ideal is to build on an existing technology, optimize the quality and completeness of reporting, and minimize additional work burdens on VA staff.

\section{Introduction}

There are currently no federal laws mandating the reporting of infectious diseases to public health authorities. Reporting requirements reside at the state level and such laws do not apply to federal agencies including the VA. Heretofore, VA's reporting of infectious diseases to public health authorities has been strictly voluntary, and has been accomplished via traditional methods (phone, mail, and fax) that are highly prone to human error, create a significant administrative burden, and do not adequately safeguard the privacy of Veterans' data. Previously, without a reporting mandate applicable to VA facilities, public health authorities have had an incomplete picture of the VA contribution to the overall infectious disease burden existing in the larger population. Moreover, at a national level, the VA has not had the ability to monitor the prevalence of the various infectious diseases within its own 151 hospitals and 827 community-based outpatient clinics. Nor has the VA been able to meet the spirit of the Health Information Technology for Clinical and Economic Health Act's Meaningful Use requirements, mandating electronic exchange of information.

\section{Methods}

The VA has developed the Healthcare Acquired Infection and Influenza Surveillance System (HAIISS), an electronic biosurveillance system. HAIISS utilizes an Application Programming Interface (API) called Medical Domain Objects to extract infectious disease data, among others, from Veterans Health Information Systems and Technology Architecture (VistA), the electronic health records system of each VA healthcare facility. The system stores the data centrally in the HAIISS Data Warehouse. HAIISS currently utilizes two software applications: QC Pathfinder and ESSENCE to monitor emerging and reportable infectious diseases or syndromes, antimicrobial utilization and drug resistance, and healthcare associated infections (HAIs) within the VA system. HAIISS has been released and is utilized by clinicians in VA medical centers and clinics in the western US, Alaska, Hawaii, and the Philippines; it will be released nationally to all inpatient and outpatient facilities of the VA.

\section{Results}

HAIISS is poised to be used for reporting infectious diseases to public health authorities. Auto-generated infectious disease and HAI reports have been built and are currently being tested. Once the data is generated by HAIISS, it can be transmitted to public health authorities in one of two ways: (1) automatically (once electronic connectivity is established with public health authorities) or (2) via mail or fax. The former has several advantages - it is real-time, eliminates underreporting, and better protects Veterans' data. The VA is currently sending syndromic surveillance data electronically to the Centers for Disease Control and Prevention's BioSense surveillance system using Health Level 7 messages. Further testing for deployment to other public health authorities is ongoing.

\section{Conclusions}

The utilization of HAIISS achieves four goals: (1) allows the VA to comply with its own Directive, as well as with pending federal legislation; (2) helps local, state, and federal public health authorities achieve a more complete picture of infectious diseases; (3) allows monitoring of infectious diseases within the VA at a national level; and (4) helps the VA comply with the Meaningful Use mandate, if additional electronic connections can be established.

\section{Keywords}

Infectious disease reporting; Meaningful Use; Department of Veterans Affairs

*Annette Lin
E-mail: Brooke.O'Hanley@va.gov 